\title{
Is the Renoprotective Effect of SGLT2 Inhibitors due to their Beneficial Effect on Hypomagnesemia?
}

\section{Tatsuo Yanagawa*}

Department of Medicine, Nerima General Hospital, Tokyo, Japan

*Corresponding author: Tatsuo Yanagawa, Department of Medicine, Nerima General Hospital, 1-24-1, Asahigaoka, Nerima-ku, Tokyo 176-8530, Japan, Tel: +81-3-5988-2200; Fax: +81-3-5988-2250; E-mail: tyana-mj@xj9.so-net.ne.jp

Received date: October 23, 2017; Accepted date: November 1, 2017; Published date: November 3, 2017

Copyright: (C) 2017 Yanagawa T. This is an open-access article distributed under the terms of the Creative Commons Attribution License, which permits unrestricted use, distribution, and reproduction in any medium, provided the original author and source are credited.

\begin{abstract}
Magnesium (Mg) deficiency is linked to diabetes and cardiovascular (CV) events. Treatment with the sodiumglucose cotransporter 2 (SGLT2) inhibitor was associated with a suppressed occurrence of CV events in patients with type 2 diabetes. We recently suggested that the increase of the serum Mg level associated with SGLT2 inhibitor treatment could explain, at least in part, the reduction of the CV events. Recently SGLT2 inhibitors have also been reported to exert a renoprotective effect and various hypotheses have been proposed regarding the mechanism. Now we would like to propose another possible mechanism, namely, the role of $\mathrm{Mg}$.
\end{abstract}

Keywords: Magnesium; Sodium-glucose cotransporter 2 inhibitor; Diabetic nephropathy; Renoprotection

\section{Magnesium Deficiency is linked to Diabetes, Hypertension and Cardiovascular Events}

Magnesium (Mg) acts as a coenzyme in the insulin secretion pathway, influences the insulin sensitivity, and acts as a modulator of $\mathrm{Na}-\mathrm{K}$ ATPase. Insufficient intake of $\mathrm{Mg}$ can cause diabetes [1] and hypertension.

$\mathrm{Mg}$ exerts favorable effects on the cardiovascular (CV) system, including an anti-inflammatory effect on the vascular endothelium and smooth muscle, vascular relaxant activity, and sympatholytic activity [2]. Of the total Mg content of the body, $99 \%$ is present within the cells, and only $1 \%$ is present outside cells. Thus, while hypomagnesemia reflects $\mathrm{Mg}$ deficiency in the whole body, $\mathrm{Mg}$ deficiency could be present even in the presence of serum $\mathrm{Mg}$ levels within the normal range [3]. Subjects with hypomagnesemia are known to be at an elevated risk of developing CV disease [4]. According to the findings of the Framingham Heart Study, hypomagnesemia is a risk factor for atrial fibrillation [5]; hypomagnesemia has also been reported to be associated with a risk of premature ventricular contractions, particularly in diabetic patients [6]. In addition, animal experiments have shown that hypomagnesemia causes reduced cardiac function [7]. Furthermore, hypomagnesemia has been reported to increase platelet aggregation, thereby facilitating thrombus formation, and to enhance coronary artery constriction, thereby increasing the risk of development of myocardial infarction [2].

A high incidence of CV events and sudden death has also been reported in patients with hypomagnesemia. A meta-analysis of relevant studies, even those including patients without diabetes, revealed that elevation of the serum $\mathrm{Mg}$ level by $0.2 \mathrm{mmol} / \mathrm{L}$ was associated with a $30 \%$ decrease in the risk of onset of $\mathrm{CV}$ disease and $40 \%$ decrease in the risk of death from CV events [8]. Adequate dietary magnesium intake has been reported to be associated with a reduced risk of cardiovascular events [9]. Thus, it might be reasonable to conclude that the risk of $\mathrm{CV}$ disease/events decreases as the serum $\mathrm{Mg}$ concentration increases.

\section{Mg Concentration Increases with SGLT2 Inhibitor Treatment}

Sodium-glucose cotransporter 2 (SGLT-2) is expressed in the proximal renal tubules, where it reabsorbs about $80-90 \%$ of the filtered glucose. Selective and reversible inhibition of SGLT2 suppresses glucose reabsorption and reduces the blood glucose levels, independent of the insulin status.

The EMPA-REG OUTCOME study revealed that treatment with the SGLT2 inhibitor empagliflozin was associated with a suppressed occurrence of $\mathrm{CV}$ events in patients with type 2 diabetes, even from an early period after the start of treatment [10]. This rapid effect of the drug is difficult to explain based on the beneficial effects of empagliflozin on the glucose and lipid metabolism alone, which are of much slower onset. Various hypotheses have been proposed, and while the precise mechanism(s) remain unknown, the ketone body hypothesis and hematocrit hypothesis have attracted the most attention [11].

Previous studies have demonstrated elevation of the serum $\mathrm{Mg}$ concentrations after the initiation of SGLT-2 inhibitor treatment [12]. We recently suggested that the increase of the serum $\mathrm{Mg}$ level associated with SGLT2 inhibitor treatment could explain, at least in part, the reduction of the $\mathrm{CV}$ risk observed in the EMPA-REG OUTCOME study [13], because, as mentioned above, hypomagnesemia increases the risk of $\mathrm{CV}$ events. We and another group reported that patients with hypomagnesemia are more likely to enjoy the beneficial effects of SGLT2 inhibitor treatment on the risk of $\mathrm{CV}$ events, because elevation of the serum $\mathrm{Mg}$ was found to be more likely to occur in patients with low serum Mg levels at the baseline $[14,15]$.

\section{Is the Renoprotective Effect of SGLT2 Inhibitors also} related to Increase of the Serum Mg Concentration? 
Page 2 of 3

SGLT2 inhibitors have also been reported to exert a renoprotective effect $[16,17]$. Several putative mechanisms have been proposed to explain this beneficial effect, such as 1) attenuation of glomerular hyperfiltration caused by the influence of the drug on the tubularglomerular feedback mechanisms [18]; 2) workload reduction of the proximal tubules and improvement of tubulointerstitial hypoxia, which allow fibroblasts to resume normal erythropoietin production [19]. Now we would like to propose another possible mechanism, namely, the role of $\mathrm{Mg}$.

In a large sample of middle-aged adults, a low total serum magnesium level was independently associated with the incidence of chronic kidney disease (CKD) and end-stage renal disease (ESRD) [20]. Hypomagnesemia is reported as a predictor of ESRD in patients with type 2 diabetic nephropathy $[21,22]$. Lower dietary magnesium intake was independently associated with greater odds of rapid kidney function decline [23]. When these findings are integrated, the renoprotective effect SGLT2 inhibitor treatment could be considered as being due to the effect of the drug in improving the serum magnesium level. However, as the above are based on observational studies, studies directly investigating the effect of $\mathrm{Mg}$ supplementation on type 2 diabetic nephropathy are warranted.

The mechanism of renal dysfunction caused by hypomagnesemia is still unclear, however, several mechanisms have been proposed (Figure 1); low magnesium levels have also been shown to promote renal vascular calcification [24]. Furthermore, serum magnesium levels may affect the endothelial function through exerting an influence on the thrombotic process [25], since low circulating magnesium levels have been shown to increase platelet aggregation and exert a prothrombotic effect. Hypomagnesemia-induced chronic inflammation [26] and hemostatic biomarkers of hypomagnesemia have been linked to athrosclerosis. An animal study indicated that $\mathrm{Mg}$ deficiency induces renal interstitial tubular injury [27]. I propose that SGLT2 inhibitor reduces kidney damage due to hypomagnesemia by elevating serum $\mathrm{Mg}$ concentration.

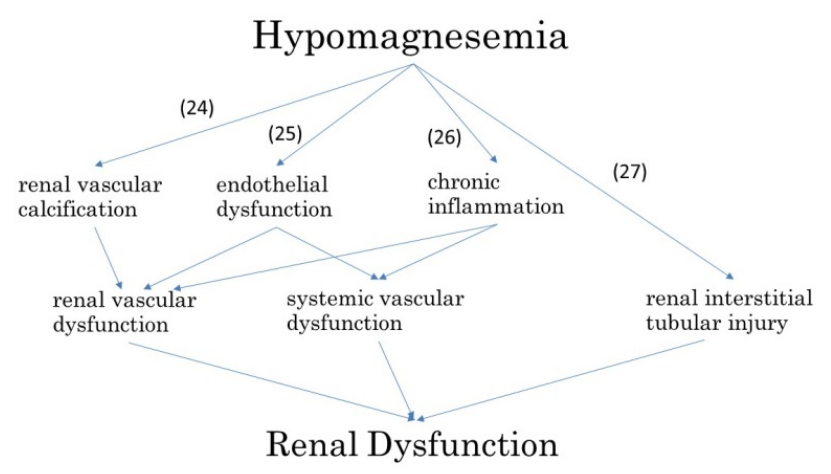

Figure 1: Hypothesis of renal dysfunction due to hypomagnesemia.

\section{Conclusion}

We would like to put forth the hypothesis that SGLT2 inhibitor therapy suppresses risk of $\mathrm{CV}$ events and exerts a renoprotective effect by improving the serum magnesium levels.

\section{References}

1. Hata A, Doi Y, Ninomiya T, Mukai N, Hirakawa Y, et al. (2013) Magnesium intake decreases Type 2 diabetes risk through the improvement of insulin resistance and inflammation: the Hisayama Study. Diabet Med 30: 1487-1494.

2. Gröber U, Schmidt J, Kisters K (2015) Magnesium in Prevention and Therapy. Nutrients 7: 8199-8226.

3. Ayuk J, Gittoes NJ (2014) Contemporary view of the clinical relevance of magnesium homeostasis. Ann Clin Biochem 51: 179-188.

4. Qu X, Jin F, Hao Y, Li H, Tang T, et al. (2013) Magnesium and the risk of cardiovascular events: a meta-analysis of prospective cohort studies. PLoS One 8: e57720.

5. Khan AM, Lubitz SA, Sullivan LM, Sun JX, Levy D, et al. (2013) Low serum magnesium and the development of atrial fibrillation in the community: the Framingham Heart Study. Circulation 127: 33-38.

6. Del Gobbo LC, Song Y, Poirier P, Dewailly E, Elin RJ, et al. (2012) Low serum magnesium concentrations are associated with a high prevalence of premature ventricular complexes in obese adults with type 2 diabetes. Cardiovasc Diabetol 11: 23.

7. Kramer JH, Spurney C, Iantorno M, Tziros C, Mak IT, et al. (2009) Neurogenic inflammation and cardiac dysfunction due to hypomagnesemia. Am J Med Sci 338: 22-27.

8. Del Gobbo LC, Imamura F, Wu JH, De Oliveira Otto MC, Chiuve SE, et al. (2013) Circulating and dietary magnesium and risk of cardiovascular disease: a systematic review and meta-analysis of prospective studies. Am J Clin Nutr 98: 160-173.

9. Guasch-Ferré M, Bulló M, Estruch R, Corella D, Martínez-González MA, et al. (2014) Dietary magnesium intake is inversely associated with mortality in adults at high cardiovascular disease risk. J Nutr 144: 55-60.

10. Zinman B, Wanner C, Lachin JM, Fitchett D, Bluhmki E, et al. (2015) Empagliflozin, Cardiovascular Outcomes, and Mortality in Type 2 Diabetes. N Engl J Med 373: 2117-2128.

11. Ferrannini E, Mark M, Mayoux E (2016) CV Protection in the EMPAREG OUTCOME Trial: A "Thrifty Substrate" Hypothesis. Diabetes Care 39: 1108-1114.

12. Tang H, Zhang X, Zhang J, Li Y, Del Gobbo LC (2016) Elevated serum magnesium associated with SGLT2 inhibitor use in type 2 diabetes patients: a meta-analysis of randomised controlled trials. Diabetologia 59: 2546-2551.

13. Yanagawa $T$ (2017) Is an Increase in Serum Magnesium One of the Causes of Cardiovascular Events Reduction in the EMPA-REG OUTCOME Study? J Clin Med Res 9: 449-450.

14. Sasaki Y, Koyano K, Iida S, Yanagawa T (2017) Mechanism Underlying Increase of the Serum Magnesium Concentration Observed Following Treatment with Sodium-Glucose Cotransporter 2 Inhibitors. J Diab Mel 7:241-248.

15. Gilbert RE, Mende C, Vijapurkar U, Sha S, Davies MJ, et al. (2017) Effects of Canagliflozin on Serum Magnesium in Patients with Type 2 Diabetes Mellitus: A Post Hoc Analysis of Randomized Controlled Trials. Diab Ther 8: 451-458.

16. Wanner $C$, Inzucchi SE, Lachin JM, Fitchett $D$, von Eynatten $M$, et al. (2016) Empagliflozin and Progression of Kidney Disease in Type 2 Diabetes. N Engl J Med 375: 323-334.

17. Neal B, Perkovic V, Mahaffey KW, de Zeeuw D, Fulcher G, et al. (2017) Canagliflozin and Cardiovascular and Renal Events in Type 2 Diabetes. N Engl J Med 377: 644-657.

18. Cherney DZ, Perkins BA, Soleymanlou N, Maione M, Lai V, et al. (2014) Renal hemodynamic effect of sodium-glucose cotransporter 2 inhibition in patients with type 1 diabetes mellitus. Circulation 129: 587-597.

19. Sano M, Takei M, Shiraishi Y, Suzuki Y (2016) Increased Hematocrit During Sodium-Glucose Cotransporter 2 Inhibitor Therapy Indicates Recovery of Tubulointerstitial Function in Diabetic Kidneys. J Clin Med Res 8: 844-847.

20. Tin A, Grams ME, Maruthur NM, Astor BC, Couper D, et al. (2015) Results from the Atherosclerosis Risk in Communities study suggest that 
Citation: Yanagawa T (2017) Is the Renoprotective Effect of SGLT2 Inhibitors due to their Beneficial Effect on Hypomagnesemia?. J Diabetes Metab 8: 772. doi:10.4172/2155-6156.1000772

Page 3 of 3

low serum magnesium is associated with incident kidney disease. Kidney Int 87: 820-827.

21. Pham PC, Pham PM, Pham PA, Pham SV, Pham HV, et al. (2005) Lower serum magnesium levels are associated with more rapid decline of renal function in patients with diabetes mellitus type 2. Clin Nephrol 63: 429-436.

22. Sakaguchi Y, Shoji T, Hayashi T, Suzuki A, Shimizu M, et al. (2012) Hypomagnesemia in type 2 diabetic nephropathy: a novel predictor of end-stage renal disease. Diabetes Care 35: 1591-1597.

23. Rebholz CM, Tin A, Liu Y, Kuczmarski MF, Evans MK, et al. (2016) Dietary Magnesium and Kidney Function Decline: The Healthy Aging in Neighborhoods of Diversity across the Life Span Study. Am J Nephrol 44: 381-387.

24. Salem S, Bruck H, Bahlmann FH, Peter M, Passlick-Deetjen J, et al. (2012) Relationship between magnesium and clinical biomarkers on inhibition of vascular calcification. Am J Nephrol 35: 31-39.
25. Dong JF, Cruz MA, Aboulfatova K, Martin C, Choi H, et al.(2008) Magnesium maintains endothelial integrity, upregulates proteolysis of ultra-large von Willebrand factor, and reduces platelet aggregation under flow conditions. Thromb Haemost 99: 586-593.

26. Maier JA, Malpuech-Brugere C, Zimowska W, Rayssiguier Y, Mazur A (2004) Low magnesium promotes endothelial cell dysfunction: implications for atherosclerosis, inflammation and thrombosis. Biochim Biophys Acta 1689: 13-21.

27. Matsuzaki H, Ohdachi J, Fuchigami M, Masuyama R, Uehara M, et al (2002) Changes in N-acetyl-beta-D-glucosaminidase activity in the urine and urinary albumin excretion in magnesium deficient rats. Biosci Biotechnol Biochem 66: 192-194. 